\title{
Primary health care provider-focused interventions for improving outcomes for people with type 2 diabetes: a rapid review
}

Nighat Faruqia ${ }^{a}$, Louise Thomas ${ }^{a}$, Sharon Parkera, Ben Harris-Roxas ${ }^{a, b}$, Jane Taggarta, Catherine Spoonera, Vincent Wong ${ }^{\mathrm{c}, \mathrm{d}}$ and Mark F Harrisª,e

${ }^{a}$ Centre for Primary Health Care and Equity, UNSW Sydney, Australia

b Primary Integrated and Community Health, South Eastern Sydney Local Health District, NSW, Australia

c South Western Sydney Clinical School, UNSW Sydney, Australia

' Diabetes and Endocrine Service, Liverpool Hospital, South Western Sydney Local Health District, NSW, Australia

e Corresponding author: m.f.harris@unsw.edu.au

\section{Article history}

Publication date: December 2019

Citation: Faruqi N, Thomas L, Parker S, HarrisRoxas B, Taggart J, Spooner C, Wong V, Harris MF. Primary health care provider-focused interventions for improving outcomes for people with type 2 diabetes: a rapid review. Public Health Res Pract. 2019;29(4):e29121903. First published: 10 April 2019. https://doi.org/10.17061/phrp29121903

\section{Key points}

- This rapid review found evidence that a variety of provider-focused primary health care interventions have positive impacts on some biochemical, clinical, psychological and health-related qualityof-life outcomes for people with type 2 diabetes

- Interventions that showed a positive impact included computerised decision support, culturally tailored programs, feedback on diabetes care quality and practice nurse involvement. The only intervention studied for which there was no evidence of benefit was decision aids, and no single intervention led to improvements in all outcomes of interest

- The challenge now is to further evaluate the applicability of these interventions in the Australian context, and to make those that are suitable for our primary health care system more widely available and improve their uptake

\section{Abstract}

Objectives and importance of the study: The bulk of care for people with type 2 diabetes occurs in primary health care. This rapid review evaluated the effectiveness of primary health care provider-focused interventions in improving biochemical, clinical, psychological and health-related quality-of-life outcomes in people with type 2 diabetes.

Methods: We searched Medline, Embase, All EBM Reviews, CINAHL, PsycINFO and grey literature focusing on the Organisation for Economic Co-operation and Development (OECD) member countries. We selected studies that targeted adults with type 2 diabetes, described a providerfocused intervention conducted in primary health care, and included an evaluation component. Four researchers extracted data and each included study was assessed for quality by two researchers.

Results: Of the 15 studies identified, there was one systematic review (high quality), four randomised controlled trials (RCTs) (two strong quality, one each moderate and weak) and 10 cluster RCTs (two strong quality, five moderate, three weak). The range of follow-up periods was 3-32 months. In all but one study, the intervention was compared against usual care. The applied interventions included: computerised and noncomputerised decision support; culturally tailored interventions; feedback to the healthcare provider on quality of diabetes care; practice nurse involvement; and integrated primary and specialist care. All interventions aimed to improve the biochemical outcomes of interest; 13 studies also included clinical, psychological and/or health-related quality-of-life outcomes. Outcome results were mixed.

Conclusions: All interventions had mixed impacts on the outcomes of interest except the one study testing a decision aid, which did not show any improvement. A number of interventions are already available in 
Australia but need wider adoption. Other effective interventions are yet to be broadly adopted, and need to be evaluated for their applicability, feasibility and sustainability in the Australian context.

\section{Introduction}

Type 2 diabetes (T2D) prevalence continues to grow in Australia, affecting an estimated $4.4 \%$ of people in 2014-15, up from 3.8\% in 2011-12. ${ }^{1}$ T2D greatly affects the person's health status and quality of life (QoL) in terms of physical, social and psychological wellbeing. Many people with T2D are not being effectively managed², which puts them at risk of developing complications. As diabetes management frequently occurs within primary health care ${ }^{3}$, many innovative interventions have been implemented involving primary health care providers (hereafter 'providers').

This brokered rapid review aimed to identify effective primary health care-based interventions that focused on capacity building, and use of technology and enablers to integrated care, and which were replicable in the Australian health environment. The research question was: Which provider-focused interventions are effective in improving clinical, biochemical, psychological and healthrelated QoL outcomes for people with T2D?

\section{Methods}

\section{Selection criteria}

Eligible studies were randomised controlled trials (RCTs), cluster RCTs or systematic reviews/metaanalyses incorporating RCT or cluster RCT designs, that evaluated effectiveness of provider-focused interventions. Studies were conducted in primary health care or in the community with primary health care providers (e.g. physicians and practice nurses), and included adult (i.e. older than 18 years) patients with T2D. We included studies that also involved patients with other diabetes types only if data on patients with T2D were reported separately. The interventions used attempted to change either individual providers' behaviour with their patients or how the providers' care was organised, to indirectly influence patient outcomes. ${ }^{4,5}$

Outcomes of interest were:

- Clinical outcomes (blood pressure [BP], and body mass index [BMI] or weight)

- Biochemical outcomes (glycosylated haemoglobin [HbA1c], lipid profile and renal function) ${ }^{3}$

- Psychological outcomes (anxiety, depression, diabetes-related distress, perceived seriousness and vulnerability, self-efficacy and self-care $)^{6}$

- Health-related QoL measures from participant self-report.
The search was limited to studies conducted in Organisation for Economic Co-operation and Development (OECD) member countries and published in English. The search for systematic reviews and metaanalyses was limited to January 2006 to June 2017, and the search for other study types to January 2012 to June 2017. Systematic reviews were excluded if more than $50 \%$ of the included studies did not evaluate one of the outcomes of interest.

\section{Search strategy}

We performed searches using five electronic databases: Medline, Embase, All EBM Reviews, CINAHL and PsycINFO. For grey literature, we searched websites in the following jurisdictions: UK/Europe (11), Australia/ New Zealand (10), US (9), Canada (7), the World Health Organization (1) and the International Diabetes Federation (1). (See supplementary file for further information: figshare.com/s/8d5042a7a401ae2600d0.)

\section{Study selection}

To assess study eligibility, one researcher reviewed each systematic review (from title and abstract), and two researchers reviewed the other study types. For selected abstracts, full-text articles were retrieved and reviewed by four researchers (with each article assessed by two researchers). At any stage of review, disagreements were resolved through team discussion.

\section{Data extraction}

Four researchers completed data extraction from included studies and entered the data into Microsoft Excel. Two other researchers conducted the grey literature search and extracted and tabulated data into a summary table.

\section{Methodological quality assessment}

Six researchers assessed the included articles for methodological quality using standardised scoring tools (with each article assessed by two researchers). ${ }^{7,8}$

\section{Results}

\section{Study selection}

From the database search we identified 15 studies that fulfilled our eligibility criteria: one systematic review, four RCTs and 10 cluster RCTs. The grey literature search did not identify any eligible studies. PRISMA flow diagrams are presented in Figures 1 and 2. 
Figure 1. PRISMA flow diagram - database search for systematic reviews

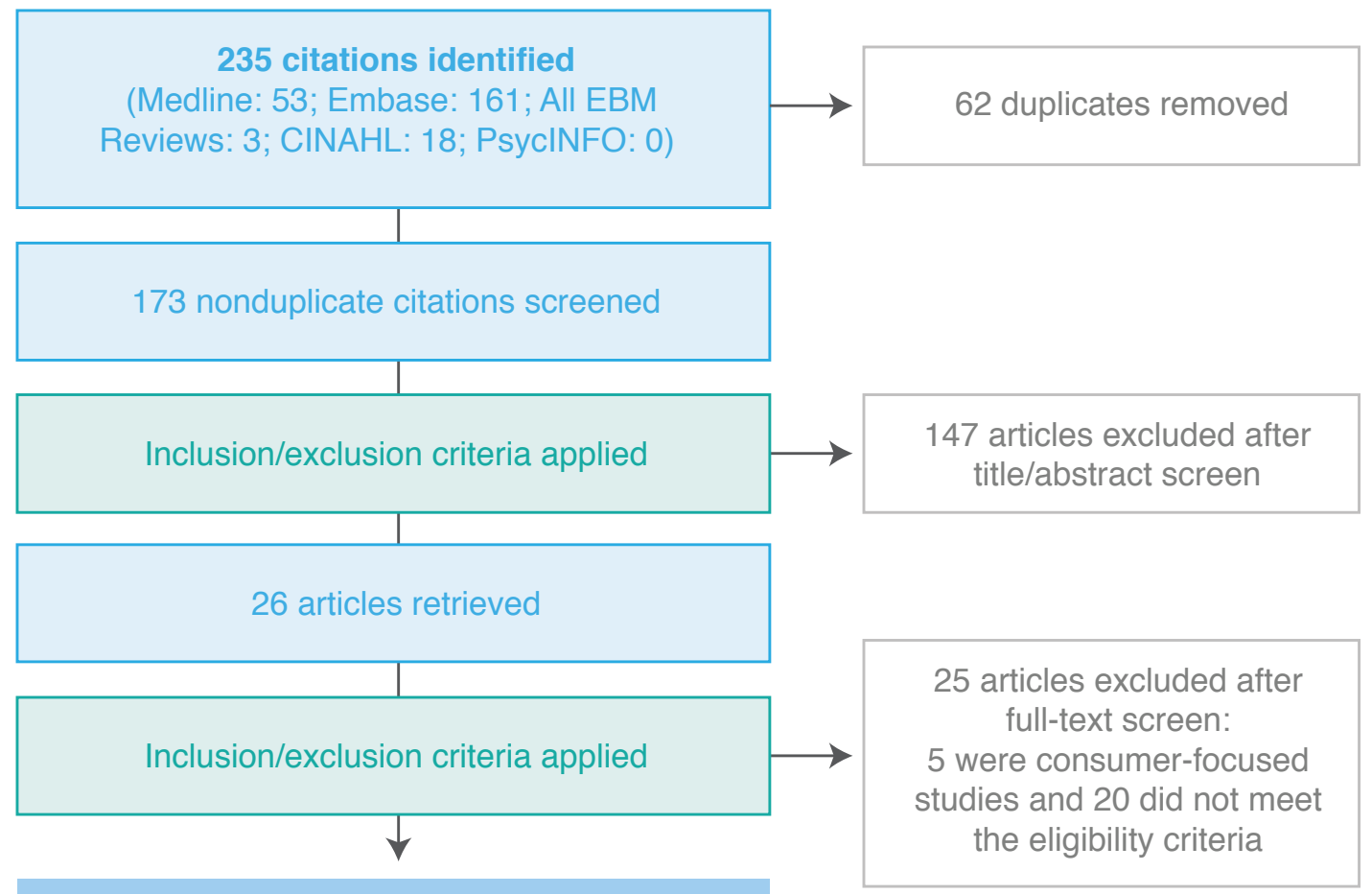

1 provider-focused study included after full-text screen

Figure 2. PRISMA flow diagram - database search for randomised controlled trials (RCTs)

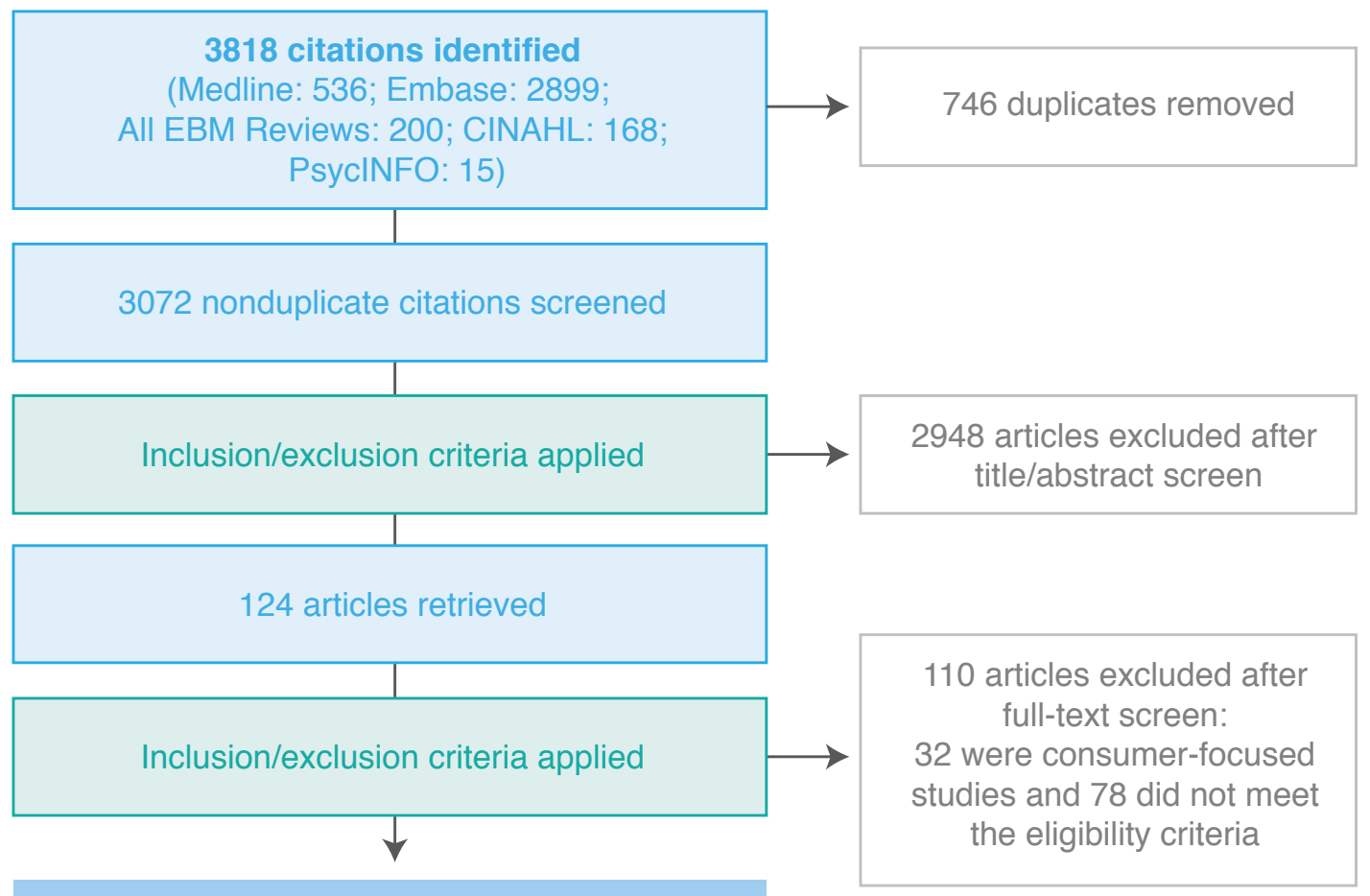

14 provider-focused studies included after full-text screen 


\section{Methodological quality assessment}

The systematic review was scored highly at $7 / 11 .{ }^{9}$ Of the four RCTs, two were of strong quality ${ }^{10,11}$, one was moderate ${ }^{12}$ and one was weak. ${ }^{13}$ Of the 10 cluster RCTs, two were of strong quality ${ }^{14,15}$, five were moderate ${ }^{16-20}$ and three were weak ${ }^{21-23}$ (Table 1).

\section{Types of interventions identified}

We classified the included studies into six categories according to the intervention applied (Table 1):

i) computerised decision-support systems (CDSS) ${ }^{9,20 \text {; }}$

ii) decision aid $^{16}$; iii) feedback on quality of diabetes care $^{14,17}$; iv) culturally tailored programs ${ }^{11,12,19}$; v) practice nurse involvement ${ }^{13,15,18,21-23}$; and vi) integrated primary health care and specialist care. ${ }^{10}$ More than half the studies included in the systematic review ${ }^{9}$ were conducted in the US. The 14 (RCT) studies were conducted in the US ${ }^{11-13,16}$, Australia ${ }^{19,21,23}$, Switzerland ${ }^{22}$, Japan ${ }^{17}$, the UK ${ }^{10}$, Europe (multicentre) $)^{14}$, Denmark ${ }^{18}$, the Netherlands ${ }^{15}$ and Spain. ${ }^{20}$ In the systematic review, the randomised patient sample sizes ranged from 62 to 7412 , and in the included RCTs from 103 to 4034.

Of the outcomes of interest, all the studies evaluated biochemical outcomes. Ten studies evaluated clinical outcomes $^{9-11,13,14,17,19,21-23}$, six evaluated psychological outcomes $^{12,13,15,18,21,23}$ and five evaluated health-related QoL outcomes. ${ }^{13,15,21-23}$

\section{Description of interventions}

\section{Computerised decision-support systems}

The only systematic review identified, by Cleveringa et al. ${ }^{9}$, reviewed 20 studies that evaluated either CDSS alone or CDSS combined with a reminder system and/or performance feedback and/or case management. They found that CDSS did not lead to improved outcomes unless combined with other interventions.

Saenz et al. ${ }^{20}$ designed and validated a simple and quick-to-use computer application to help providers make decisions about insulin therapy. The intervention group obtained statistically significant $\mathrm{HbA} 1 \mathrm{c}$ reductions compared to the control patients, although a significant proportion of patients did not reach a target $\mathrm{HbA1c}$ level of less than $7.0 \%$.

\section{Decision aids}

Branda et al. ${ }^{16}$ compared decision aids administered to patients for starting statins or choosing antihyperglycaemic agents against usual care, after providing minimal training to the clinicians. The study found no significant impact on patients' biochemical measures.

\section{Feedback on quality of diabetes care}

In two cluster RCTs ${ }^{14,17}$, benchmarking data were collected prospectively and provided to clinicians to inform their treatment planning. In one of these, the feedback group demonstrated a significantly greater HbA1c improvement than the control group. ${ }^{17}$

\section{Culturally tailored programs}

Three studies evaluated culturally tailored programs that involved intensively trained community health workers, and which were aimed at overcoming linguistic and cultural barriers to providing appropriate/adequate care. McDermott et al. ${ }^{19}$ evaluated a case-management approach to the care of Indigenous adults in remote Australia, while studies in the US focused on the Latino population. ${ }^{11,12}$ The Australian study and one of the US studies ${ }^{11,19}$ achieved improved HbA1c levels; the other US study ${ }^{12}$ achieved significant improvements in health status, and depression and anxiety symptoms.

\section{Practice nurse involvement}

One RCT and five cluster RCTs involved nurses. In Australia ${ }^{23}$, a trial involved practice nurses taking on an enhanced role in managing insulin dosing in liaison with general practitioners. The practice nurses, mentored by a diabetes educator, attended a training session of 6090 minutes. Patients showed improved $\mathrm{HbA} 1 \mathrm{c}$ and mental health. Another Australian study ${ }^{21}$ evaluated structured telephone coaching by practice nurses, who received 2 days of training. At follow-up, the effect on biochemical and clinical outcomes did not differ significantly between intervention and control groups.

Frei et al. ${ }^{22}$ investigated whether a team-based approach including a trained practice nurse would improve patients' cardiovascular risk profiles. The training included a 6-day course that empowered practice nurses for their role in the team. Although no improvement was seen in $\mathrm{HbA1C}$, BP and low-density lipoprotein (LDL) cholesterol did improve.

Gabbay et al. ${ }^{13}$ trained practice nurses in motivational interviewing and behaviour-change counselling. After the intervention, they found systolic BP and depression symptom scores were better among the intervention group compared to the control group. Juul et al. ${ }^{18}$ evaluated the effectiveness of nurses undergoing a 16-hour self-determination, theory-based course, which included communication training, delivered over 10 months. The study found no effect on patient HbA1c compared with usual practice, although patients' cholesterol levels did improve. In another study ${ }^{15}$, practice nurses attended three 8-hour training sessions, followed by booster sessions, to integrate biopsychosocial selfmanagement support into routine consultations. No significant differences were seen between the intervention and control arms for any outcomes. 
Table 1. Summary characteristics of included studies by intervention type

\begin{tabular}{|c|c|c|c|c|c|c|c|c|c|c|}
\hline \multirow[b]{2}{*}{$\begin{array}{l}\text { Intervention } \\
\text { category }\end{array}$} & \multirow[b]{2}{*}{$\begin{array}{l}\text { Study } \\
\text { (year) }\end{array}$} & \multirow[b]{2}{*}{$\begin{array}{l}\text { Study type (quality } \\
\text { assessment) }\end{array}$} & \multirow{2}{*}{$\begin{array}{l}\text { Provider } \\
\text { type (total } \\
\text { providers } n \text { ) }\end{array}$} & \multirow{2}{*}{$\begin{array}{l}\text { Total } \\
\text { patients } \\
(n)\end{array}$} & \multirow[b]{2}{*}{$\begin{array}{l}\text { Follow-up } \\
\text { (months) }\end{array}$} & \multirow[b]{2}{*}{ Control } & \multirow[b]{2}{*}{ Intervention } & \multicolumn{3}{|c|}{ Study outcomes ${ }^{a}$} \\
\hline & & & & & & & & Measures & $\begin{array}{l}\text { Results } \\
(95 \% \mathrm{Cl})\end{array}$ & $\begin{array}{c}p \\
\text { value }^{b}\end{array}$ \\
\hline \multirow[t]{7}{*}{$\begin{array}{l}\text { Computerised } \\
\text { decision- } \\
\text { support } \\
\text { systems }\end{array}$} & \multirow[t]{7}{*}{$\begin{array}{l}\text { Cleveringa } \\
\text { et al. } \\
(2013)^{9}\end{array}$} & \multirow[t]{7}{*}{$\begin{array}{l}\text { Systematic review } \\
\text { of } 20 \text { studies } \\
(7 / 11)\end{array}$} & \multirow[t]{7}{*}{ NSp } & \multirow[t]{7}{*}{ 62-7412 } & \multirow[t]{7}{*}{$6-32$} & \multirow[t]{7}{*}{$\begin{array}{l}\text { Usual } \\
\text { care }\end{array}$} & \multirow[t]{7}{*}{$\begin{array}{l}\text { CDSS only }\left(4^{c}\right) \\
\text { CDSS in combination with: } \\
\text { (i) reminders }\left(5^{c}\right) \\
\text { (ii) performance feedback }\left(3^{c}\right) \\
\text { (iii) case management or case } \\
\text { management + reminders }\left(2^{c}\right) \\
\text { (iv) reminders + performance } \\
\text { feedback }\left(4^{c}\right) \\
\text { (v) reminders, performance } \\
\text { feedback + case } \\
\text { management }\left(2^{c}\right)\end{array}$} & $\begin{array}{c}\text { CDSS onlyd: } \\
\text { HbA1c (0/3); lipids } \\
\text { (0/2); BP (0/2) (in } \\
\text { 1 study SBP increased } \\
\text { significantly in the } \\
\text { intervention arm) } \\
\text { CDSS in combination: } \\
\text { (i) HbA1c (0/1); LDL-C } \\
\text { (0/1); SBP (0/1) }\end{array}$ & & \\
\hline & & & & & & & & $\begin{array}{l}\text { (ii) } \operatorname{HbA1c}(1 / 2) \\
\text { lipids (0/2); } \operatorname{SBP}(0 / 1)\end{array}$ & & 0.009 \\
\hline & & & & & & & & $\begin{array}{c}\text { (iii) } \operatorname{HbA} 1 \mathrm{c}(2 / 2) ; \mathrm{SBP} \\
(1 / 1)\end{array}$ & & $<0.01$ \\
\hline & & & & & & & & $\begin{array}{c}\text { TC (1/1); HDL-C (1/1); } \\
\text { percentage achieving } \\
\text { HbA1c < } 7 \% \text { target }\end{array}$ & & $<0.05$ \\
\hline & & & & & & & & $\begin{array}{c}\text { (iv) } \\
\text { HbA1c (3/4) } \\
\operatorname{SBP}(0 / 3) ; \mathrm{DBP}(0 / 2) ; \\
\operatorname{LDL}-\mathrm{C}(0 / 2)\end{array}$ & & 0.01 \\
\hline & & & & & & & & $\begin{array}{c}(\mathrm{v}) \\
\text { Composite of HbA1c } \\
<7+\mathrm{SBP}<130+\mathrm{LDL}-\mathrm{C} \\
<100 \mathrm{mg} / \mathrm{dL}(2 / 2) \\
\mathrm{HbA1c}(0 / 1)\end{array}$ & & 0.002 \\
\hline & & & & & & & & $\begin{array}{c}\text { SBP; DBP; TC; LDL-C; } \\
\text { composite of HbA1C } \\
<7+\text { SBP }<140+\text { LDL-C } \\
<2.5(1 / 1)\end{array}$ & & $<0.05$ \\
\hline
\end{tabular}


Table 1. Summary characteristics of included studies by intervention type (continued)

\begin{tabular}{|c|c|c|c|c|c|c|c|c|c|c|}
\hline \multirow[b]{2}{*}{$\begin{array}{l}\text { Intervention } \\
\text { category }\end{array}$} & \multirow[b]{2}{*}{$\begin{array}{l}\text { Study } \\
\text { (year) }\end{array}$} & \multirow[b]{2}{*}{$\begin{array}{l}\text { Study type (quality } \\
\text { assessment) }\end{array}$} & \multirow{2}{*}{$\begin{array}{l}\text { Provider } \\
\text { type (total } \\
\text { providers } n \text { ) }\end{array}$} & \multirow{2}{*}{$\begin{array}{l}\text { Total } \\
\text { patients } \\
(n)\end{array}$} & \multirow[b]{2}{*}{$\begin{array}{l}\text { Follow-up } \\
\text { (months) }\end{array}$} & \multirow[b]{2}{*}{ Control } & \multirow[b]{2}{*}{ Intervention } & \multicolumn{3}{|c|}{ Study outcomes ${ }^{a}$} \\
\hline & & & & & & & & Measures & $\begin{array}{l}\text { Results } \\
(95 \% \mathrm{Cl})\end{array}$ & $\begin{array}{c}p \\
\text { value }^{b}\end{array}$ \\
\hline Decision aids & $\begin{array}{l}\text { Branda et al. } \\
(2013)^{16}\end{array}$ & $\begin{array}{c}\text { Cluster RCT } \\
\text { (moderate) }\end{array}$ & $\begin{array}{l}\text { Physicians, } \\
\text { nurses, } \\
\text { physician } \\
\text { assistants } \\
\text { (NSp) }\end{array}$ & 103 & 9 & $\begin{array}{l}\text { Usual } \\
\text { care }\end{array}$ & $\begin{array}{l}\text { Clinicians implemented the } \\
\text { Diabetes Medication Choice } \\
\text { decision aid and usual care } \\
\text { statin discussion or the Statin } \\
\text { Choice decision aid and usual } \\
\text { care for antihyperglycaemic } \\
\text { medications discussion }\end{array}$ & $\begin{array}{l}\text { HbA1c; } \\
\text { LDL-C }\end{array}$ & & NS \\
\hline \multirow[t]{6}{*}{$\begin{array}{l}\text { Feedback } \\
\text { on quality of } \\
\text { diabetes care }\end{array}$} & \multirow[t]{3}{*}{$\begin{array}{l}\text { Hayashino } \\
\text { et al. } \\
(2016)^{17}\end{array}$} & \multirow[t]{3}{*}{$\begin{array}{l}\text { Cluster RCT } \\
\text { (moderate) }\end{array}$} & \multirow[t]{3}{*}{$\begin{array}{l}\text { Primary care } \\
\text { physicians } \\
\text { (192) }\end{array}$} & \multirow[t]{3}{*}{2199} & \multirow[t]{3}{*}{12} & \multirow[t]{3}{*}{$\begin{array}{l}\text { Usual } \\
\text { care }\end{array}$} & \multirow{3}{*}{$\begin{array}{l}\text { Physicians received a monthly } \\
\text { report on care quality. Patients } \\
\text { received reminders for physician } \\
\text { visits and lifestyle modifications }\end{array}$} & $\mathrm{HbA} 1 \mathrm{c}^{e}$ & $\begin{array}{l}-0.14 \\
(-0.26 \\
-0.02)\end{array}$ & 0.027 \\
\hline & & & & & & & & BMle & $\begin{array}{l}-0.21 \\
(-0.33 \\
-0.98)\end{array}$ & 0.002 \\
\hline & & & & & & & & SBP; DBP & & NS \\
\hline & \multirow{3}{*}{$\begin{array}{l}\text { Hermans } \\
\text { et al. } \\
(2013)^{14}\end{array}$} & \multirow{3}{*}{$\begin{array}{l}\text { Cluster RCT } \\
\text { (strong) }\end{array}$} & \multirow{3}{*}{$\begin{array}{l}\text { Primary care } \\
\text { physicians } \\
(477)\end{array}$} & \multirow{3}{*}{3996} & \multirow{3}{*}{12} & \multirow{3}{*}{$\begin{array}{l}\text { Usual } \\
\text { care }\end{array}$} & \multirow{3}{*}{$\begin{array}{l}\text { Standard care with feedback } \\
\text { benchmarked against } \\
\text { colleagues }\end{array}$} & LDL-C & $\begin{array}{l}54.3 \text { vs } \\
49.7\end{array}$ & 0.006 \\
\hline & & & & & & & & $\mathrm{HbA} 1 \mathrm{C}+\mathrm{SBP}+\mathrm{LDL}-\mathrm{C}$ & 12.5 vs 8.1 & $<0.001$ \\
\hline & & & & & & & & $\mathrm{HbA} 1 \mathrm{c}$ & & NS \\
\hline
\end{tabular}


Table 1. Summary characteristics of included studies by intervention type (continued)

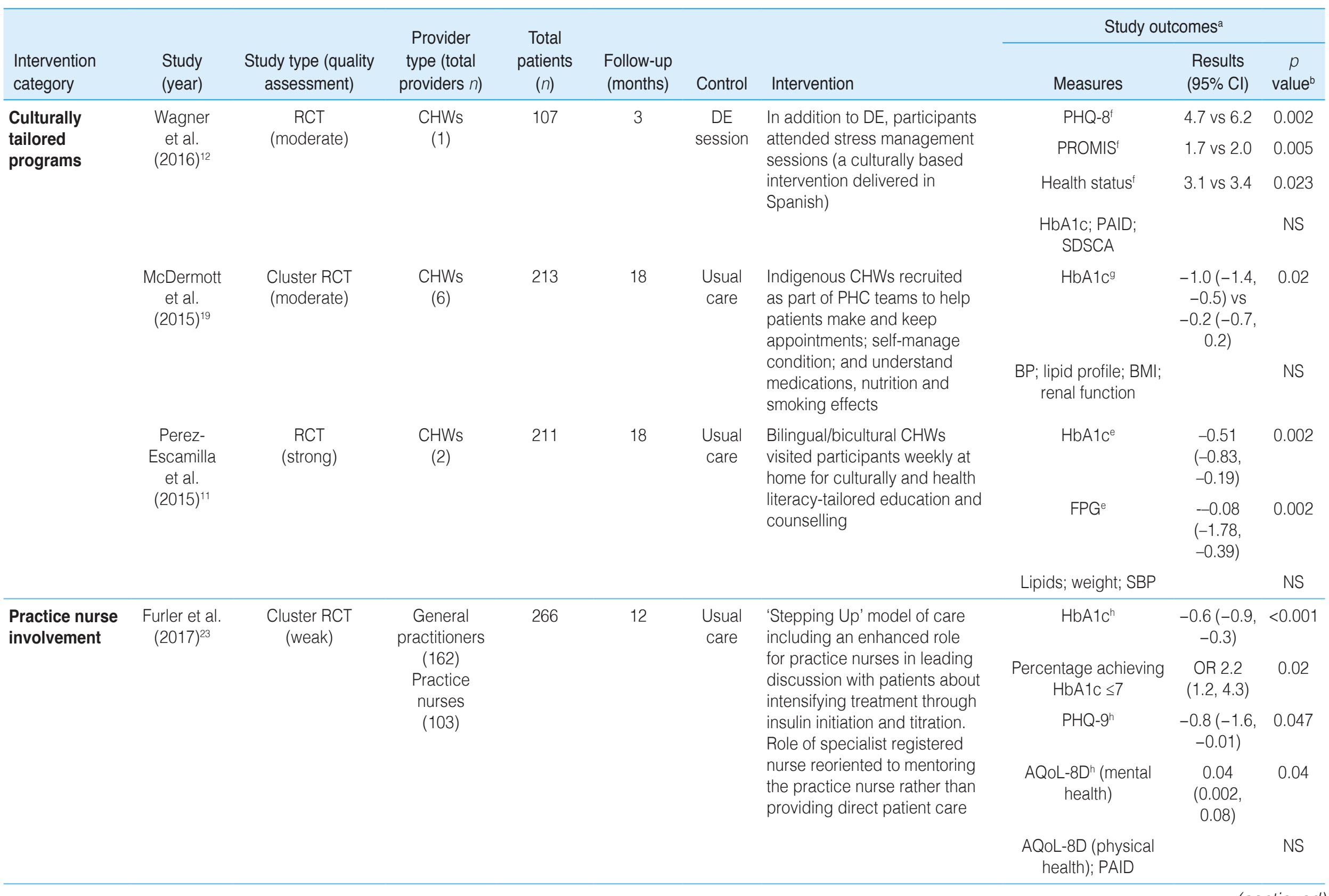


Table 1. Summary characteristics of included studies by intervention type (continued)

\begin{tabular}{|c|c|c|c|c|c|c|c|c|c|c|}
\hline \multirow[b]{2}{*}{$\begin{array}{l}\text { Intervention } \\
\text { category }\end{array}$} & \multirow[b]{2}{*}{$\begin{array}{l}\text { Study } \\
\text { (year) }\end{array}$} & \multirow[b]{2}{*}{$\begin{array}{l}\text { Study type (quality } \\
\text { assessment) }\end{array}$} & \multirow{2}{*}{$\begin{array}{l}\text { Provider } \\
\text { type (total } \\
\text { providers } n \text { ) }\end{array}$} & \multirow{2}{*}{$\begin{array}{l}\text { Total } \\
\text { patients } \\
(n)\end{array}$} & \multirow[b]{2}{*}{$\begin{array}{l}\text { Follow-up } \\
\text { (months) }\end{array}$} & \multirow[b]{2}{*}{ Control } & \multirow[b]{2}{*}{ Intervention } & \multicolumn{3}{|c|}{ Study outcomes } \\
\hline & & & & & & & & Measures & $\begin{array}{l}\text { Results } \\
(95 \% \mathrm{Cl})\end{array}$ & $\begin{array}{c}p \\
\text { value }^{b}\end{array}$ \\
\hline \multirow[t]{9}{*}{$\begin{array}{l}\text { Practice nurse } \\
\text { involvement, } \\
\text { continued }\end{array}$} & $\begin{array}{l}\text { van Dijk- } \\
\text { de Vries } \\
\text { et al. } \\
(2015)^{15}\end{array}$ & $\begin{array}{l}\text { Cluster RCT } \\
\text { (strong) }\end{array}$ & $\begin{array}{l}\text { Practice } \\
\text { nurses } \\
(40)\end{array}$ & 264 & 12 & $\begin{array}{l}\text { Usual } \\
\text { care }\end{array}$ & $\begin{array}{l}\text { Practice nurses trained to integrate } \\
\text { detection and follow-up phase } \\
\text { of self-management support into } \\
\text { daily practice. Training followed by } \\
\text { booster sessions }\end{array}$ & $\begin{array}{l}\text { HbA1c; PAID; SF12; } \\
\text { self-efficacy }\end{array}$ & & NS \\
\hline & \multirow[t]{4}{*}{$\begin{array}{l}\text { Frei et al. } \\
(2014)^{22}\end{array}$} & \multirow[t]{4}{*}{$\begin{array}{l}\text { Cluster RCT } \\
\text { (weak) }\end{array}$} & \multirow[t]{4}{*}{$\begin{array}{l}\text { Practice } \\
\text { nurses } \\
\text { (NSp) }\end{array}$} & \multirow[t]{4}{*}{326} & \multirow[t]{4}{*}{12} & \multirow[t]{4}{*}{$\begin{array}{l}\text { Usual } \\
\text { care }\end{array}$} & \multirow{4}{*}{$\begin{array}{l}\text { Implementation of CCM elements } \\
\text { via a trained practice nurse: } \\
\text { organisation of healthcare and } \\
\text { delivery system design; clinical } \\
\text { information systems (using } \\
\text { a monitoring tool); decision } \\
\text { support (with guideline-based } \\
\text { instructions on the monitoring } \\
\text { tool and requiring the availability } \\
\text { of a diabetes specialist); and } \\
\text { self-management support }\end{array}$} & $\mathrm{SBPh}$ & $\begin{array}{c}-3.63 \\
(-7.26 \\
0.00)\end{array}$ & 0.050 \\
\hline & & & & & & & & $\mathrm{DBP}^{\mathrm{h}}$ & $\begin{array}{l}-4.01 \\
(-6.23 \\
11.78)\end{array}$ & $<0.001$ \\
\hline & & & & & & & & LDL-C ${ }^{h}$ & $\begin{array}{l}-0.21 \\
(-0.39 \\
-0.02)\end{array}$ & 0.033 \\
\hline & & & & & & & & $\mathrm{HbA} 1 \mathrm{c}^{\mathrm{h}}$ & & NS \\
\hline & \multirow[t]{2}{*}{$\begin{array}{l}\text { Juul et al. } \\
(2014)^{18}\end{array}$} & \multirow[t]{2}{*}{$\begin{array}{l}\text { Cluster RCT } \\
\text { (moderate) }\end{array}$} & \multirow{2}{*}{$\begin{array}{c}\text { Practice } \\
\text { nurses }(1-5 / \\
\text { each, } \\
40 \text { practices })\end{array}$} & \multirow[t]{2}{*}{4034} & \multirow[t]{2}{*}{18} & \multirow[t]{2}{*}{$\begin{array}{l}\text { Usual } \\
\text { care }\end{array}$} & \multirow{2}{*}{$\begin{array}{l}\text { Over } 10 \text { months, practice } \\
\text { nurses received a 16-hour } \\
\text { course which met the SDT- } \\
\text { based recommendations on } \\
\text { provider behaviour including } \\
\text { communication training }\end{array}$} & $\mathrm{TC}^{\mathrm{e}}$ & $\begin{array}{c}0.08(0.01 \\
0.15)\end{array}$ & 0.02 \\
\hline & & & & & & & & HbA1c; PAID; SF12 & & NS \\
\hline & \multirow[t]{2}{*}{$\begin{array}{l}\text { Gabbay } \\
\text { et al. } \\
(2013)^{13}\end{array}$} & \multirow[t]{2}{*}{$\begin{array}{c}\mathrm{RCT} \\
\text { (weak) }\end{array}$} & \multirow[t]{2}{*}{$\begin{array}{l}\text { Nurse case } \\
\text { managers } \\
\text { (3) }\end{array}$} & \multirow[t]{2}{*}{545} & \multirow[t]{2}{*}{24} & \multirow[t]{2}{*}{$\begin{array}{l}\text { Usual } \\
\text { care }\end{array}$} & $\begin{array}{l}\text { Bilingual and bicultural nurse } \\
\text { case managers received } \\
\text { motivational interview training }\end{array}$ & $\mathrm{SBP}^{\dagger}$ & 131 vs 135 & 0.045 \\
\hline & & & & & & & $\begin{array}{l}\text { and provided motivational } \\
\text { interview-guided behaviour- } \\
\text { change counselling. Visits, } \\
\text { conducted in language of choice, } \\
\text { included review of test results, } \\
\text { lifestyle behaviour, medication } \\
\text { adherence and complications } \\
\text { screening. Referrals made } \\
\text { and primary care physicians } \\
\text { prompted for medication } \\
\text { titrations, when necessary }\end{array}$ & $\begin{array}{c}\text { HbA1c; } \\
\text { LDL-C; DBP }\end{array}$ & 10 vs 14 & 0.02 \\
\hline
\end{tabular}


Table 1. Summary characteristics of included studies by intervention type (continued)

\begin{tabular}{|c|c|c|c|c|c|c|c|c|c|c|}
\hline \multirow[b]{2}{*}{$\begin{array}{l}\text { Intervention } \\
\text { category }\end{array}$} & \multirow[b]{2}{*}{$\begin{array}{l}\text { Study } \\
\text { (year) }\end{array}$} & \multirow[b]{2}{*}{$\begin{array}{l}\text { Study type (quality } \\
\text { assessment) }\end{array}$} & \multirow{2}{*}{$\begin{array}{l}\text { Provider } \\
\text { type (total } \\
\text { providers } n \text { ) }\end{array}$} & \multirow{2}{*}{$\begin{array}{c}\text { Total } \\
\text { patients } \\
(n)\end{array}$} & \multirow[b]{2}{*}{$\begin{array}{c}\text { Follow-up } \\
\text { (months) }\end{array}$} & \multirow[b]{2}{*}{ Control } & \multirow[b]{2}{*}{ Intervention } & \multicolumn{3}{|c|}{ Study outcomes ${ }^{a}$} \\
\hline & & & & & & & & Measures & $\begin{array}{c}\text { Results } \\
(95 \% \mathrm{Cl})\end{array}$ & $\begin{array}{c}p \\
\text { value }^{b}\end{array}$ \\
\hline $\begin{array}{l}\text { Practice nurse } \\
\text { involvement, } \\
\text { continued }\end{array}$ & $\begin{array}{c}\text { Blackberry } \\
\text { et al. } \\
(2013)^{21}\end{array}$ & $\begin{array}{l}\text { Cluster RCT } \\
\quad \text { (weak) }\end{array}$ & $\begin{array}{l}\text { Practice } \\
\text { nurses } \\
(70)\end{array}$ & 473 & 18 & $\begin{array}{l}\text { Usual } \\
\text { care }\end{array}$ & $\begin{array}{l}\text { Practice nurses taught to } \\
\text { deliver telephone coaching on } \\
\text { self-management, and trained } \\
\text { to engage patients through } \\
\text { scheduled and structured } \\
\text { telephone sessions on lifestyle } \\
\text { issues, medication adherence } \\
\text { and dosing, self-monitoring, } \\
\text { and taking greater initiative } \\
\text { in therapeutic alliance with } \\
\text { the treating doctor, facilitating } \\
\text { appropriate intensification } \\
\text { of medications to achieve } \\
\text { treatment goals }\end{array}$ & $\begin{array}{c}\text { HbA1c; lipids; renal } \\
\text { function; weight; BMl; } \\
\text { BP; self-efficacy; AQoL; } \\
\text { PHQ-9 }\end{array}$ & & NS \\
\hline $\begin{array}{l}\text { Integrated } \\
\text { primary health } \\
\text { care and } \\
\text { specialist care }\end{array}$ & $\begin{array}{l}\text { Basudev } \\
\text { et al. } \\
(2016)^{10}\end{array}$ & $\begin{array}{c}\mathrm{RCT} \\
\text { (strong) }\end{array}$ & $\begin{array}{l}\text { Specialist } \\
\text { nurses, } \\
\text { diabetologists, } \\
\text { general } \\
\text { practitioners } \\
\text { (NSp) }\end{array}$ & 208 & 12 & $\begin{array}{l}\text { Usual } \\
\text { care }\end{array}$ & $\begin{array}{l}\text { Exposure to a 'virtual clinic', } \\
\text { which included clinical review, } \\
\text { assessment of clinical needs and } \\
\text { therapy review, management } \\
\text { plan formulation, and a follow-up } \\
\text { patient consultation to agree on } \\
\text { a care plan }\end{array}$ & $\begin{array}{c}\text { SBPg } \\
\text { HbA1c; DBP; TC; } \\
\text { weight; BMI; eGFR }\end{array}$ & $\begin{array}{c}-6 \pm 16 \text { vs } \\
2 \pm 18\end{array}$ & $\begin{array}{l}0.008 \\
\text { NS }\end{array}$ \\
\hline
\end{tabular}

$\mathrm{AQoL}=$ Assessment of Quality of Life; BMI = body mass index; BP = blood pressure; CA = computer application; CCM = chronic care model; CDSS = computerised decision-support system; CES-D = Center for Epidemiologic Studies Depression; CHW = community health worker; $\mathrm{Cl}=$ confidence interval; $\mathrm{DBP}=$ diastolic blood pressure; $\mathrm{DE}=$ diabetes education; $\mathrm{eGFR}=$ estimated glomerular filtration rate; FPG = fasting plasma glucose; HbA1c = glycosylated haemoglobin; HDL-C = high-density lipoprotein cholesterol; LDL-C = low-density lipoprotein cholesterol; NS = not significant; NSp = not specified; OR = odds ratio; PAID = Problem Areas in Diabetes; PHQ = Patient Health Questionnaire; PHC = primary health care; PROMIS = Patient-Reported Outcomes Measurement Information System; RCT = randomised controlled trial; SBP = systolic blood pressure; SDSCA = Summary of Diabetes Self-Care Activities; SDT = Self-determination theory; SF = Short

Form: $\mathrm{TC}=$ total cholesterol

a Of interest only

b As reported in papers

c Number of included studies

d In parenthesis, numerator indicates studies with significant outcomes out of the total studies reporting that variable

e Follow-up group difference, mean (with or without 95\% Cl)

Follow-up means (control vs intervention)

g Baseline and follow-up difference (95\% Cl or \pm SD)

n Between-group baseline to follow-up difference, mean (95\% Cl)

Note: Unless specified otherwise, units for each measure are BMI: kg/m²; BP: mmHg; FPG: mmol/L; HbA1c: \%; lipids: mmol/L; weight: kg. 


\section{Integrated primary health care and specialist} care

Basudev et al. ${ }^{10}$ evaluated a model of integrated primary health care and specialist care, based on a 'virtual clinic', which was intended to enhance clinical expertise among primary health care providers. The intervention group showed better control of BP than the control group.

\section{Discussion}

The shift of T2D clinical care from specialist to primary care requires additional support for primary health care providers to deliver appropriate management. This is especially important in rural regions in which specialised services are less accessible than in urban areas. ${ }^{24}$ This rapid review evaluated which (if any) interventions targeted at primary health care providers led to positive changes in outcomes in patients with T2D. We identified 15 studies (one systematic review, four RCTs and 10 cluster RCTs) of varying quality with risk of bias identified, which used six different types of interventions. All interventions had mixed impacts on the outcomes of interest, except the one study testing a decision aid, which did not result in any improvement.

Our study identified mixed evidence for improved outcomes in response to interventions provided in primary care involving CDSS, feedback on performance, enhanced roles for practice nurses, integrated care and culturally tailored programs.

Feedback on performance and CDSS could be readily applied in the Australian context, given the high level of computerisation and the existence of evidence-based guidelines for diabetes management in primary health care. ${ }^{3,25}$ However, this is hampered by poor connectivity with specialist services and concern about potential distortions caused by performance-based funding mechanisms. ${ }^{26}$ The development of the Australian Government's My Health Record initiative and shared electronic systems for care planning provide opportunities for these types of interventions to be more widely and systematically implemented. ${ }^{27}$

Greater practice nurse involvement in diabetes care is consistent with how practice nurse roles are currently construed and defined. However, involvement of practice nurses is hindered by a number of factors, including general practitioners' negative attitudes towards practice nurses having a greater role in patient management, competing demands on nurse time, lack of space and restrictive practice nurse job descriptions. ${ }^{28,29}$ There is also a need for continuing professional development of practice nurses, especially in dietary assessment and advice, and intensification of medication management. ${ }^{23,30}$ However, the six studies we identified in which practice nurses had an enhanced role in T2D management and received specific training showed mixed results, with improvements in some but not all parameters.

Australia is culturally diverse, yet many ethnic groups experience socio-economic disadvantage and higher levels of health risk. ${ }^{31}$ Aboriginal and Torres Strait Islander Australians in particular experience high rates of T2D. ${ }^{2}$ The culturally tailored programs that we reviewed involved community health workers, who have well-established roles in diabetes care overseas, delivering education or selfmanagement support. In Australia, Aboriginal health workers have increasing roles in supporting chronic disease selfmanagement ${ }^{32}$; however, community health workers have not been systematically involved in the primary care of other culturally and linguistically diverse groups. While consistent with the objectives of the Australian National Diabetes Strategy 2016-2020, the involvement of community health workers is not specifically mentioned in the strategy. ${ }^{33}$ The widespread adoption of a community health worker role in diabetes care in Australia requires clear policy direction to overcome professional barriers. It also requires investment and implementation by Local Health Networks, Primary Health Networks and nongovernment organisations.

\section{Limitations}

This review identified only six types of interventions. Other interventions might exist but were not included according to the selection criteria. The results on the effectiveness of interventions might also change when trialled in different settings and with different population groups for longer periods of time. Furthermore, we were interested in surrogate outcomes and hence did not identify long-term clinical outcomes such as development of cardiovascular disease.

\section{Conclusions}

We found evidence that computerised decision support, culturally tailored programs, feedback on diabetes care quality, and practice nurse involvement have the potential to exert a positive influence on some biochemical, clinical, psychological and health-related QoL outcomes in patients with T2D. One study involving an intervention that aimed to integrate primary health care and specialist care showed some evidence of improvement in patients' blood pressure, but not other outcomes.

Of the various effective provider-focused interventions we identified, some are already part of the current Australian primary health care environment - for example, the provision of feedback to general practitioners on quality of diabetes care, and the role of practice nurses in diabetes care. The challenge, however, is to make these interventions more widely available and improve their uptake. Evidence-based interventions that are yet to be broadly adopted include culturally tailored programs involving community health workers that target immigrant populations, and diabetes decision support incorporated into practice software. For these interventions, research is needed to further evaluate their applicability, feasibility and sustainability in the Australian context. 


\section{Acknowledgement}

This work is based on an Evidence Check review brokered by the Sax Institute for the NSW Agency for Clinical Innovation.

\section{Peer review and provenance}

Externally peer reviewed, not commissioned.

\section{Competing interests}

$\mathrm{MH}$ is a board member of the Central and Eastern Sydney Primary Health Network.

\section{Author contributions}

NF and LT were responsible for the design of the review, analysis of data and drafting and editing of the manuscript. SP contributed to the design, study review and manuscript editing. $\mathrm{BH}-\mathrm{R}$ contributed to the design, analysis of data, study review and manuscript editing. JT and CS contributed to the design and were responsible for study review and manuscript editing. VW provided clinical advice and reviewed and edited the manuscript. $\mathrm{MH}$ was responsible for the design of the review, interpretation of findings and drafting and editing of the manuscript.

\section{References}

1. Australian Bureau of Statistics. National health survey: first results, 2014-15. Canberra: ABS; 2015 [cited 2018 May 8]. Available from: www.abs.gov.au/ausstats/ abs@.nsf/Lookup/by\%20Subject/4364.0.55.001 201415 Main\%2OFeatures About\%20the\%20National\%20 Health\%20Survey 3

2. Australian Bureau of Statistics. Australian health survey: biomedical results for chronic diseases, 2011-12. Canberra: ABS; 2013. [cited 2018 May 13]. Available from: www.abs.gov.au/AUSSTATS/abs@.nsf/ DetailsPage/4364.0.55.0052011-12

3. The Royal Australian College of General Practitioners. General practice management of type 2 diabetes: 2016-18. Melbourne: RACGP; 2016. [cited 2018 May 6]. Available from: static. diabetesaustralia.com.au/s/ fileassets/diabetes-australia/5d3298b2-abf3-487e-9d5e0558566fc242.pdf

4. Conn VS, Ruppar TM, Enriquez M, Cooper PS, Chan KC. Healthcare provider targeted interventions to improve medication adherence: systematic review and metaanalysis. Int J Clin Pract. 2015;69(8):889-99.

5. Oncology Nursing Society. Healthcare provider interventions. Pittsburgh, PA: Oncology Nursing Society [cited 2018 May 30]. Available from: www.ons.org/node/5301
6. Thoolen B, Gorter K, De Ridder D, Rutten G, Bensing J. Psychological outcomes of patients with screendetected type 2 diabetes. The influence of time since diagnosis and treatment intensity. Diabetes Care. 2006;29(10):2257-62.

7. Shea B, Hamel C, Wells G, Bouter L, Kristjansson E, Grimshaw J, et al. AMSTAR is a reliable and valid measurement tool to assess the methodological quality of systematic reviews. J Clin Epidemiol. 2009;62(10):1013-20.

8. Higgins J, Green S (eds). Cochrane handbook for systematic reviews of interventions: version 5.1.0 (updated March 2011). West Sussex: The Cochrane Collaboration; 2011 [cited 2019 Jan 17]. Available from: handbook-5-1.cochrane.org/

9. Cleveringa FG, Gorter KJ, van den Donk M, van Gijsel J, Rutten GE. Computerized decision support systems in primary care for type 2 diabetes patients only improve patients' outcomes when combined with feedback on performance and case management: a systematic review. Diabetes Technol Ther. 2013;15(2):180-92.

10. Basudev N, Crosby-Nwaobi R, Thomas S, Chamley M, Murrells T, Forbes A. A prospective randomized controlled study of a virtual clinic integrating primary and specialist care for patients with Type 2 diabetes mellitus. Diabetic Med. 2016;33(6):768-76.

11. Perez-Escamilla R, Damio G, Chhabra J, Fernandez ML, Segura-Perez S, Vega-Lopez S, et al. Impact of a community health workers-led structured program on blood glucose control among Latinos with type 2 diabetes: The DIALBEST Trial. Diabetes Care. 2015;38(2):197-205.

12. Wagner JA, Bermudez-Millan A, Damio G, SeguraPerez S, Chhabra J, Vergara C, et al. A randomized, controlled trial of a stress management intervention for Latinos with type 2 diabetes delivered by community health workers: outcomes for psychological wellbeing, glycemic control, and cortisol. Diabetes Res Clin Pract. 2016;120:162-70.

13. Gabbay RA, Anel-Tiangco RM, Dellasega C, Mauger DT, Adelman A, Van Horn DHA. Diabetes nurse case management and motivational interviewing for change (DYNAMIC): Results of a 2-year randomized controlled pragmatic trial. J Diabetes. 2013;5(3):349-57.

14. Hermans MP, Elisaf M, Michel G, Muls E, Nobels F, Vandenberghe $\mathrm{H}$, et al. Benchmarking is associated with improved quality of care in type 2 diabetes: the OPTIMISE randomized, controlled trial. Diabetes Care. 2013;36(11):3388-95.

15. van Dijk-de Vries A, Bokhoven MA, Winkens B, Terluin B, Knottnerus JA, Van Der Weijden T, et al. Lessons learnt from a cluster-randomised trial evaluating the effectiveness of Self-Management Support (SMS) delivered by practice nurses in routine diabetes care. BMJ Open. 2015;5(6):e007014. 
16. Branda ME, LeBlanc A, Shah ND, Tiedje K, Ruud K, Van Houten $\mathrm{H}$, et al. Shared decision making for patients with type 2 diabetes: a randomized trial in primary care. BMC Health Serv Res. 2013;13:301.

17. Hayashino Y, Suzuki H, Yamazaki K, Goto A, Izumi K, Noda M. A cluster randomized trial on the effect of a multifaceted intervention improved the technical quality of diabetes care by primary care physicians: The Japan Diabetes Outcome Intervention Trial-2 (J-DOIT2). Diabetic Med. 2016;33(5):599-608.

18. Juul L, Maindal HT, Zoffmann V, Frydenberg M, Sandbaek A. Effectiveness of a training course for general practice nurses in motivation support in type 2 diabetes care: a cluster-randomised trial. PLoS ONE. 2014;9(5):e96683.

19. McDermott RA, Schmidt B, Preece C, Owens V, Taylor S, $\mathrm{Li} \mathrm{M}$, et al. Community health workers improve diabetes care in remote Australian Indigenous communities: results of a pragmatic cluster randomized controlled trial. BMC Health Serv Res. 2015;15:68.

20. Saenz A, Brito M, Moron I, Torralba A, Garcia-Sanz E, Redondo J. Development and validation of a computer application to aid the physician's decision-making process at the start of and during treatment with insulin in type 2 diabetes: a randomized and controlled trial. J Diabetes Sci Technol. 2012;6(3):581-88.

21. Blackberry ID, Furler JS, Best JD, Chondros P, Vale M, Walker C, et al. Effectiveness of general practice based, practice nurse led telephone coaching on glycaemic control of type 2 diabetes: the Patient Engagement and Coaching for Health (PEACH) pragmatic cluster randomised controlled trial. BMJ. 2013;347:f5272.

22. Frei A, Senn O, Chmiel C, Reissner J, Held U, Rosemann T. Implementation of the chronic care model in small medical practices improves cardiovascular risk but not glycemic control. Diabetes Care. 2014;37(4):1039-47.

23. Furler J, O'Neal D, Speight J, Manski-Nankervis JA, Gorelik A, Holmes-Truscott E, et al. Supporting insulin initiation in type 2 diabetes in primary care: results of the Stepping Up pragmatic cluster randomised controlled clinical trial. BMJ. 2017;356:j783.

24. Skinner T, Allen P, Peach E, Browne J, Pouwer F, Speight J, et al. Does the shortage of diabetes specialists in regional and rural Australia matter? Results from Diabetes MILES_-Australia. Diabetes Res Clin Pract. 2013;100(2):222-29.
25. Schoen C, Osborn R, Doty M, Squires D, Peugh J, Applebaum S. A survey of primary care physicians in eleven countries, 2009: Perspectives on care, costs, and experiences. Health Aff. 2009;28:w1171-83.

26. Wright M. Pay-for-performance programs: do they improve the quality of primary care? Aust Fam Physician. 2012;41(12):989-91.

27. Australian Government. Canberra: Australian Digital Health Agency. My health record Australian Government [cited 2018 Jun 6]. Available from: www.myhealthrecord.gov.au

28. Halcomb E, Salamonson Y, Davidson P, Kaur R, Young S. The evolution of nursing in Australian general practice: a comparative analysis of workforce surveys ten years on. BMC Fam Pract. 2014;15:52.

29. Stephen C, Mclnnes S, Halcomb E. The feasibility and acceptability of nurse-led chronic disease management interventions in primary care: An integrative review. J Adv Nurs. 2018;74(2):279-88.

30. Hollis M, Glaister K, Lapsley J. Do practice nurses have the knowledge to provide diabetes self-management education? Contemp Nurse. 2014;46:234-41.

31. Australian Institute of Health and Welfare: Thow A, Waters A-M. Diabetes in culturally and linguistically diverse Australians: identification of communities at high risk. Canberra: AlHW; 2005 [cited 2018 Apr 24]. Available from: www.aihw.gov.au/getmedia/ebe47968-5b5a-47febb7c-2f695770e7d8/dclda.pdf.aspx?inline=true

32. Stoneman A, Atkinson D, Davey M, Marley J. Quality improvement in practice: improving diabetes care and patient outcomes in Aboriginal Community Controlled Health Services. BMC Health Serv Res. 2014;14:481.

33. Australian Government Department of Health. Australian National Diabetes Strategy 2016-2020. Canberra: Commonwealth of Australia; 2015. [cited 2018 Jun 6]. Available from: www.health.gov.au/internet/main/ publishing.nsf/content/3AF935DA210DA043CA257EFB0 00D0C03/\$File/Australian\%20National\%20Diabetes\%20 Strategy\%202016-2020.pdf

\section{Copyright: (C) $\underset{\text { BY (P) }(2)}{\text { SA }}$}

(c) 2019 Faruqi et al. This article is licensed under the Creative Commons Attribution-NonCommercial-ShareAlike 4.0 International Licence, which allows others to redistribute, adapt and share this work non-commercially provided they attribute the work and any adapted version of it is distributed under the same Creative Commons licence terms. See: www.creativecommons.org/licenses/by-nc-sa/4.0/ 